\title{
Review \\ Clinical review: Critical care transport and austere critical care
} David H Rice, George Kotti and William Beninati

\author{
Uniformed Services University of the Health Sciences, 2200 Bergquist Drive, Suite \#1, Wilford Hall Medical Center, Lackland Air Force Base, \\ TX 78236, USA
}

The views expressed herein are those of the authors and do not reflect US Air Force or US Department of Defense policy.

Corresponding author: William Beninati, william.beninati@lackland.af.mil

Published: 5 March 2008

Critical Care 2008, 12:207 (doi:10.1186/cc6782)

This article is online at http://ccforum.com/content/12/2/207

(C) 2008 BioMed Central Ltd

\begin{abstract}
The development of modern intensive care units (ICUs) has allowed the survival of patients with advanced illness and injury, although at a cost of substantial infrastructure. Natural disasters and military operations are two common situations that can create critically ill patients in an environment that is austere or has been rendered austere. This has driven the development of two related strategies to care for these casualties. Portable ICU capability can be rapidly established in the area of need, providing relatively advanced capability but limited capacity and sustainability. The other strategy is to rapidly evacuate critically ill and injured patients following their initial stabilization. This permits medical personnel in the austere location to focus resources on a larger number of less critical patients. It also permits the most vulnerable patients to receive care in an advanced center. This strategy requires careful planning to overcome the constraints of the transport environment. The optimal strategy has not been determined, but a combination of these two approaches has been used in recent disasters and military operations and is promising. The critical care delivered in an austere setting must be integrated with a long-term plan to provide follow-on care.
\end{abstract}

\section{Introduction}

The capability to provide medical care to critically ill patients has evolved considerably over the last half-century. Hospitals developed intensive care units (ICUs) where special expertise and equipment could be used for unstable patients. Recent studies have elucidated the significant impact on patient outcomes that result from ICU physician staffing models [1]. Data suggesting that the intensity of ICU staffing alone can affect a change in overall hospital morality and length of stay for critically ill populations lend credence to the importance of adequate and aggressive ICU care. As the field of critical care develops, it has become clear not only that ICUs are effective tools for resuscitation and stabilization of the critically ill, but that the skill with which the treatments are initiated there have lasting effects on the overall hospital course of the patient. A modern ICU represents a complex assembly of skilled personnel and physical infrastructure. This infrastructure must include space to support patients and staff; temperature control; secure oxygen, electricity, water, and vacuum sources; medical supplies; pharmaceutical agents; and equipment [2]. ICUs also have ready access to surgical, radiographic, transfusion, and laboratory capabilities.

The level of care available in an ICU establishes a standard of care for unstable patients. Natural disasters and human conflict are two common occurrences leading caregivers to develop the capability to extend this standard of care into austere environments. Natural disasters may strike population centers with advanced medical care, simultaneously producing casualties and incapacitating even a well-developed health care system $[3,4]$. Disasters also can strike remote regions with little pre-existing medical infrastructure. The sudden increase in the number of critically ill patients following a disaster can be overwhelming, and caregivers in this setting face major challenges in establishing a critical care capability [5]. Human conflict also has the potential to create casualties and destroy or incapacitate a health care system and often adds security to the caregiver's concerns. In response to these challenges, two major strategies have emerged: portable critical care and critical care transport. A working group of the Society of Critical Care Medicine has considered the situation in which an infrastructure is intact but overwhelming numbers of casualties occur, such as in a bioterrorist attack. This group has developed the concept of augmenting critical care in place [6]. There has been significant development in austere critical care but, to date, little structured scientific study. This review examines what has been learned about provision of critical care in such austere environments.

$\mathrm{CCATT}=$ Critical Care Air Transport Team; EMEDS = Expeditionary Medical System; ICP = intracranial pressure; ICU = intensive care unit; IMSURT = International Medical-Surgical Response Team; USAF = US Air Force. 


\section{Critical care in disasters - recent experience}

The medical response to recent disasters illustrates how critical care was successfully provided within the disaster area. A major earthquake struck western Turkey in August 1999, resulting in thousands of casualties and major damage to the region's medical infrastructure. The Israeli Defense Forces deployed a field hospital to the city of Adapazari, where 2,627 people died and 5,084 were wounded [7]. This hospital included a 12-bed ICU in which they managed 63 patients. The ICU was staffed with 3 physicians, 3 nurses, and 5 paramedics. Over the course of 2 weeks, this team managed a range of medical, trauma, and post-surgical patients. To enhance their sustainability, they successfully integrated with the local medical system to augment their equipment and supplies. One of the major functions cited in the report was preparation of patients for transfer to unaffected areas.

In June 2001, Houston, Texas was struck by Tropical Storm Allison, causing major flooding. This resulted in closure or major curtailment of services in nine hospitals with resultant compromise in emergency and critical care in the city. As part of the US federal response, the US Air Force (USAF) deployed a 25-bed portable field hospital from Wilford Hall Medical Center in San Antonio, Texas. This facility was operational with 3.5 hours of arrival in a non-medical structure and, during an 11-day stay, successfully cared for 1,036 patients, including 33 ICU patients [8]. Lives were saved in this facility, and it validated the model of military response with a portable hospital/ICU for disasters within the US.

In December 2003, an earthquake struck Bam, Iran, causing many thousands of casualties and disabling the city's medical system. There was a brisk international response, with many nations deploying field hospitals to assist. A US-based team reported on their experience operating a portable field hospital, with an ICU, in Bam [9]. The authors faced a range of casualties from those suffering acute trauma to delayed complications such as soft tissue infection and compartment syndrome as well as exacerbation of chronic illness. This report describes the hardship from operating continuously with little infrastructure and emphasizes that medical capability is ineffective without non-medical infrastructure such as communication, safety, sanitation, and security. They emphasize the critical role of casualty evacuation outside of the disaster area.

\section{Critical care in field hospitals - general considerations}

Field hospitals have been developed by military medical services, civilian governments, and non-governmental organizations to serve the population affected by war, unrest, or disaster. From a critical care perspective, these hospitals must prepare not only to address trauma or direct effects of a disaster, but also to treat pre-existing disease and decompensation of patients with comorbid conditions. The surgical and critical care capabilities of a medical center cannot be duplicated in a matter of hours or days, but portable, rapidly deployable teams have been developed to provide the major components of resuscitation and stabilization. Considering the design characteristics of an ideal fixed ICU, a deployable ICU can approach this with some conscious compromises. In general, enhancing capability, capacity, or sustainability increases cost and complexity and decreases portability.

The capability that can be developed with prior planning and investment stands in contrast to the situation that confronts health care workers forced to improvise after disaster destroys their resources. Between disasters, limited resources and pressing daily needs force hospitals to de-emphasize disaster preparedness [5]. In September 2005, Hurricane Katrina devastated the US Gulf Coast, basic infrastructure was destroyed, and the extensive health care system across this region was severely curtailed. Disaster response planners established Louis Armstrong International Airport as a primary evacuation site for the city of New Orleans. Initial responders were overwhelmed by the high number of patients and lack of basic infrastructure such as potable water, medical supplies, and communications with command authorities. A primitive field hospital with ICU capabilities was established at the airport. These providers were hampered by an immediate lack of ICU resources such as ventilators, oxygen, and respiratory therapists. Additionally, there was no mechanism for resupply or patient evacuation [10]. One of the authors (DHR) participated in the response as a member of a USAF Critical Care Air Transport Team (CCATT) tasked to evacuate critically ill patients from the New Orleans airport. His team primarily managed critical illness resulting from destruction of the existing medical infrastructure rather than direct storm damage. Their evacuees included patients with recent liverkidney transplant, acute upper gastrointestinal bleeding in a setting of cirrhosis, critical hyperkalemia in dialysis-dependent renal failure, and delirium tremens in an alcoholic. As would be expected in an overwhelmed medical system, these patients were consuming the attention of their caregivers to the exclusion of non-critical patients. The lack of a stockpile of simple portable mechanical ventilators meant that some patients in respiratory failure were bag-ventilated by hand for many hours, completely occupying a caregiver each.

An easily overlooked complication of critical care in austere locations is infection. The challenge of maintaining infection control in an improvised ICU includes control of environmental contamination from the exterior as well as the risk of cross-contamination between patients in crowded conditions and when basic supplies are limited. In addition, organisms particular to that environment may be encountered. Tsunami victims evacuated from Southeast Asia in 2004 exhibited highly resistant strains of less common organisms. The difficult-to-treat Acinetobacter, Pseudomonas, and Stenotrophomonas cultured from the evacuees not only placed 
them at increased risk, but also exposed native patients to the organisms once they were introduced into the existing health care system [11]. Among the casualties in Iraq, locally acquired Acinetobacter baumannii infection has been a major challenge [12]. A recent report indicates that factors of austere environment and native organisms can be successfully managed. In a small series, the authors report on their experience with definitive repair of open facial fractures in critically injured patients while in a tent hospital, without causing serious wound infections [13].

\section{Critical care augmentation}

\section{Expanding/providing critical care on scene}

During the Cold War, the US military prepared for massive engagement in a predictable location and medical capability was developed accordingly. Post-Cold War, the US military has been heavily employed in a spectrum of operations from disaster relief, humanitarian, and peacekeeping operations through war. Medical capability has kept pace through development of deployable field hospital systems. The USAF system, termed Expeditionary Medical System (EMEDS), is designed with modules that interlock to build to an advanced hospital when fully deployed. The initial module, termed the Small Portable Expeditionary Aeromedical Rapid Response (SPEARR) team, consists of $10 \mathrm{crew}$ members with equipment that can fit within a medium-size trailer. The modest size makes this team readily deployable. This unit is highly capable, providing primary care and public health support, general and orthopedic surgery, and a critical care resuscitation and holding capability, although capacity and unsupported sustainability are limited. As a given operation matures and the requirement for capacity increases, modules can be added to create a 10- or 25-bed hospital with an ICU capability. Specialty modules, including ICU, can be added to this backbone depending on mission requirements. The hospital modules can also be linked to create a theater hospital, which is essentially a field medical center. The EMEDS is housed in a tent system with climate control that allows it to function across a range of temperature extremes. Table 1 lists the major ICU-support equipment items used by EMEDS. The full range of configurations for EMEDS has been used to support current coalition military operations in Iraq and Afghanistan. A 60-bed EMEDS theater hospital operating at Balad Air Base, Iraq, provides comprehensive care for a large volume of coalition military and Iraqi civilian casualties.

A major benefit of EMEDS is that capability assigned to US bases, when not deployed overseas, provides a surge capacity within the US in the event of disaster. An EMEDS was used effectively in this capacity when Tropical Storm Allison caused flooding in Houston, Texas, as noted above [4]. A US civilian team with capability similar to a basic EMEDS is the International Medical-Surgical Response Team (IMSURT) based in Boston, Massachusetts. This capability has been used effectively to care for rescue workers at the
World Trade Center following the attack on September 11, 2001; victims of a nightclub fire in Rhode Island; and for earthquake victims in Iran [7,14]. The EMEDS and IMSURT provide a model of how hospital systems can develop the capability to provide trauma/critical care surge capacity or preliminary replacement capability if their location is rendered austere by disaster.

The current EMEDS theater hospital in Balad, Iraq, is the 332nd Expeditionary Medical Group/Air Force Theater Hospital. It is the primary evacuation hub for all injured coalition casualties in Iraq. This hospital currently functions on the model of a US Level I trauma center and provides continuous coverage by trauma surgeons, critical care physicians, and other surgical subspecialists. It is currently comprised of $18 \mathrm{ICU}$ beds, 10 emergency room bays, 2 computed tomography scanners, and 4 surgical suites running up to 8 operating tables. During the period from 1 January to 31 December 2005, 4,700 patients were admitted and approximately one quarter of all admissions required intensive care with more than 5,000 ventilator days and 9,000 operative procedures performed during this time [15].

The major limitations of these systems are sustainability and capacity. A central consideration in establishing this capability is the ability of the local heath care system to absorb the follow-on care initiated at these facilities. Planning for this follow-on care needs to incorporate the local medical system and possibly non-governmental organizations that provide and develop medical capability in austere locations. As local follow-on care is being developed, austere critical care must include a robust mechanism for patient transport to locations where they can be absorbed and where the resuscitative measures initiated in the austere environment can be continued.

\section{Relieving area of critical care load}

Transport of unstable patients away from an austere location relieves a load on the local resources. However, moving an unstable patient exposes that patient to risk. Within a hospital, this risk is weighed each time an ICU patient is moved for a diagnostic or therapeutic procedure. Long-range transport adds a significant degree of difficulty to all of the risks from in-hospital transport. The potential complications of transport include accidentally dislodging life-sustaining devices, diverting attention from physiologic trends to attend to the transport, temporarily suspending access to needed capability, and the chance of a mishap directly related to the transport [16]. Guidelines for the transport of critically ill adults have been published [17]. General principles of critical care transport include ensuring that the move is in the patient's best interest, development of a pre-transport plan that meets the patient's ongoing and anticipated needs with no decrease in level of care, and execution of the plan by a well-trained, well-equipped team. 
Table 1

Major equipment items used by US Air Force Expeditionary Medical System to provide critical care

Medications
Antibiotics
Amphotericin
Metronidazole
Gentamicin
Ceftriaxone
Vancomycin
Ampicillin/Sulbactam
Piperacillin/Tazobactam
Levofloxacin
Cefazolin
Acyclovir
Bacitracin

Vasopressors/Inotropes/Antiarrhythmics

Phenylephrine

Norepinephrine

Digoxin

Dopamine

Procainamide

Dobutamine

Adenosine

Lidocaine

Sedatives/Analgesics/Paralytics

Haloperidol

Fentanyl

Acetaminophen

Ibuprofen

Midazolam

Propofol

Ketorolac

Morphine sulfate

Meperidine

Lorazepam

Diazepam

Phenobarbitol

Etomidate

Succinylcholine

Vecuronium

Cardiac/Antihypertensives

Nitroglycerine tablets, paste, and drip

Nitroprusside

Aspirin

Heparin

Verapamil

Labetalol

Metoprolol

Alteplase

Tenecteplase

Furosemide

Phentolamine

Isoproterenol

Diltiazem

\section{Medications}

Pulmonary medications

Albuterol sulfate metered dose inhaler (MDI) and liquid Ipratropium bromide MDI and liquid

Racepinephrine

Levalbuterol MDI

Toxicology

Acetylcysteine

Charcoal-activated suspension

Flumazenil

Protamine

Naloxone

Glucagon

Miscellaneous

Mannitol

Promethazine

Metoclopramide

Cimetidine

Ranitidine

Potassium chloride

Magnesium sulfate

Diphenhydramine

Sodium polystyrene

Advanced cardiac life support (ACLS)

All medications specified to under ACLS protocol

Blood products

Packed red blood cells

Fresh frozen plasma

Factor VIla

Whole blood (platelets not widely available) (donors are pre-screened)

Laboratory

Access to Expeditionary Medical System laboratory

Shelter

Alaska Structures Medical Shelter (tent system)

(Alaska Structures, Inc., Anchorage, AK, USA)

Electricity

Generated on site with MEP-7 electrical generator

Oxygen

Generated on site with EDOCS 120B oxygen generator

Diagnostic imaging

Access to Expeditionary Medical System radiology

(includes computed tomography in some cases)

Major equipment items

Impact 754 ventilator (Impact Instrumentation, Inc., West Caldwell, NJ, USA)

Pulmonetics LTV 1000 ventilator (Pulmonetic Systems, Inc., Minneapolis, MN, USA)

Welch Allyn Propaq 206 EL physiologic monitor (Welch Allyn, Skaneateles, NY, USA)

Zoll CCT monitor/defibrillator/external pacemaker (ZOLL Medical Corporation, Chelmsford, MA, USA)

Pentax FB15-X bronchoscope (Pentax Instruments, Tokyo, Japan) 


\section{US Air Force Critical Care Air Transport Teams}

The post-Cold War increase in scope and complexity of military operations drove the need for an agile medical system that could deploy and redeploy rapidly and scale to fit the requirements. At the same time, concepts of damage control surgery for trauma patients were developed, offering a strategy for enhanced survival of combat casualties. The military required teams that could deploy close to combat units to provide lifesaving surgical resuscitation, but teams capable of providing post-resuscitation care were too large and complex to keep pace with the movements of the forces they supported $[18,19]$. In response to this situation, the USAF developed Critical Care Aeromedical Transport Teams (CCATTs) to provide the existing aeromedical evacuation system with an intrinsic capability to rapidly evacuate critical casualties with no decrease in the level of care, allowing the forward surgical units to prepare for the next round of casualties [20]. This paradigm shift has been employed in the wars in Iraq and Afghanistan. Comparison of mortality data between military conflicts is difficult due to changes in weapons, tactics, and personal protection; nevertheless, survival of combat casualties has been strikingly improved over prior wars and this improvement may be due in part to this system for delivering advanced care [19].

A CCATT is composed of a physician trained in a critical care-related field, a critical care nurse, and a respiratory therapist. For purposes of critical care transport, the USAF employs physicians from pulmonary/medical critical care, cardiology, anesthesia, emergency medicine, and surgical critical care. In addition to being fully trained and current in their specialties, each member participates in a 2-week basic aeromedical training program with follow-on training exercises. Training is composed of didactic lessons focusing on the stresses of flight, team supplies and equipment, and lessons learned from previous missions. Teams then participate in a number of simulated scenarios using patient simulators and actual aircraft models with the goal of experiencing firsthand the challenges inherent in providing critical care in austere conditions. At intervals, re-currency training is offered in a cooperative program at the University of Cincinnati Medical Center (Cincinnati, $\mathrm{OH}, \mathrm{USA}$ ) which includes a review of content from the initial course along with direct patient care. The teams are equipped with a standardized backpack set that contains equipment and supplies to care for three critically ill patients. Table 2 lists the major equipment items. Missions are performed on large military cargo aircraft reconfigured for medical care, although smaller aircraft or helicopters are sometimes used. A trained crew can convert the interior of a cargo aircraft for medical use within minutes, providing for efficient use of these aircraft. The aircraft currently used most often is the C-17 Globemaster III, which is well lit and has built-in and easily accessible systems for medical oxygen and electricity. The C-17 has a primary function of cargo and troop transport but can be configured in the field to accommodate 36 litter and
54 ambulatory patients and attendants. It has a speed of 450 knots at an altitude of 28,000 feet. The $\mathrm{C}-17$ has an unrefueled range of approximately 2,400 nautical miles and unlimited range with aerial refueling. Of particular usefulness for aeromedical evacuation is the aircraft's ability to operate at small airfields. The C-17 can take off and land on runways as short as 3,500 feet and as wide as only 90 feet $[21,22]$.

The duration of CCATT missions ranges from 1 to more than 18 hours. The teams operate as a component of the comprehensive USAF Aeromedical Evacuation System that coordinates all phases of transport and provides nurse-based crews with advanced training for in-flight operations and safety. During the period of January 2005 through August 2006, this system performed 40,410 patient movements globally, of which 1,203 were managed by CCATTs (US Transportation Command).

The scope of care for a CCATT is designed to match that of a typical ICU. Ideally, the patients, while critically ill, have undergone an initial assessment and appropriate resuscitation. In some instances, as occurred during the Hurricane Katrina evacuation, initial stabilization is performed by CCATTs. Most patients referred to CCATT require mechanical ventilation; central-venous, arterial, and intracranial pressure (ICP) monitors are often employed; and patients are frequently receiving vasoactive and sedative infusions. The nursing ratio of $1: 3$ is less than usually occurs in groundbased ICUs, but for physicians and respiratory therapists the ratio is significantly greater than usual. This has led to a pattern of teamwork and cross-functioning which has proven to be highly effective.

\section{Challenges in long-range critical care air transport}

Large cargo aircraft offer major advantages as ICU-transport vehicles. They cover long distances rapidly, can move multiple patients simultaneously, and provide more space than smaller platforms. However, the environment of an aircraft in flight presents significant challenges. In contrast to a hospital, an aircraft cabin experiences rapid shifts in barometric pressure and has a marked reduction in relative humidity. Significant acceleration occurs during takeoff, landing, turbulence, and the tactical maneuvering that is required during certain military flights. Workspace is constricted and access to all parts of the patient is sometimes compromised. Accessing electrical power often requires special equipment to convert typical $400-\mathrm{Hz}$ aircraft power to 50 to $60 \mathrm{~Hz}$. Total available amperage, limited by aircraft design, may be insufficient for some medical configurations. Oxygen supplies are generally limited, so the team must perform a pre-flight calculation of oxygen requirements and supplies, including a safety factor that accounts for the likelihood of a change in patient status or flight plan. Supplies, medications, and equipment are limited to what is carried aboard, so it is important to carefully 
Table 2

Major equipment items used by US Air Force Critical Care Air Transport Teams to provide critical care in flight

\begin{tabular}{|c|c|}
\hline Aircraft used & Medications \\
\hline C-17, C-130, KC-135, C-9, C-21, others as available/required & Cardiac/Antihypertensives \\
\hline Electricity & Phentolamine \\
\hline Converted from aircraft electrical system & Isoproterenol \\
\hline Oxygen & Diltiazem \\
\hline Built-in aircraft oxygen system (C-17); portable liquid or & Toxicology \\
\hline compressed oxygen on others & Flumazenil \\
\hline Medications & Naloxone \\
\hline Antibiotics & Glucagon \\
\hline Vancomycin & Pulmonary medications \\
\hline Cefazolin & Albuterol sulfate metered dose inhaler (MDI) \\
\hline Ceftriaxone & Ipratropium bromide MDI \\
\hline Clindamycin & Racepinephrine \\
\hline Gentamycin & Miscellaneous \\
\hline Levofloxicin & Mannitol \\
\hline Amoxicillin/Clavulanate & Albunim \\
\hline Ampicillin/Sulbactam & Promethazine \\
\hline Piperacillin/Tazobactam & Metoclopramide \\
\hline Vasopressors/Inotropes/Antiarrhythmics & Cimetidine \\
\hline Dopamine & Ranitidine \\
\hline Dobutamine & Potassium chloride \\
\hline Lidocaine & Magnesium sulfate \\
\hline Epinephrine & Diphenhydramine \\
\hline Noepinephrine & Sodium polystyrene \\
\hline Vasopressin & Phenytoin \\
\hline Phenylephrine & Methylprednisolone \\
\hline Digoxin & Dexamethasone \\
\hline Adenosine & Advanced cardiac life support (ACLS) \\
\hline Procainamide & All medications specified to under ACLS protocol \\
\hline Amiodarone & Supplies \\
\hline Sedatives/Analgesics/Paralytics & Crystalloid and colloid IV fluid and tubing \\
\hline Propofol & Central venous and arterial access kits \\
\hline Midazolam & Chest tube insertion kits and pluerovax \\
\hline Lorazepam & Nasopharyngeal, oral, laryngeal mask, and endotrachial airways \\
\hline Haloperidol & with intubation supplies \\
\hline Etomidate & Cricothyrotomy kits \\
\hline Meperidine & Portable intracranial pressure monitor \\
\hline Morphine sulfate & Foley catheters \\
\hline Fentanyl & Sterile gowns and gloves \\
\hline Acetaminophen & Wound dressing supplies \\
\hline Ketorolac & Ventilator circuits, heat/moisture exchangers, and $\mathrm{EtCO}_{2}$ \\
\hline Vecronium & (end-tidal carbon dioxide) monitoring supplies \\
\hline Succinylcholine & Laboratory \\
\hline Cardiac/Antihypertensives & i-STAT blood analyzer (i-STAT Corporation, East Windsor, NJ, USA) \\
\hline Nitroglycerine spray and IV & Major equipment items \\
\hline Nitroprusside & Three Impact 754 ventilators (Impact Instrumentation, Inc., \\
\hline Aspirin & West Caldwell, NJ, USA) \\
\hline Heparin & $\begin{array}{l}\text { Three Welch Allyn Propaq } 206 \text { EL physiologic monitors } \\
\text { (Welch Allyn, Skaneateles, NY, USA) }\end{array}$ \\
\hline Enoxaparin & One Zoll CCT monitor/defibrillator/external pacemaker \\
\hline Verapamil & (ZOLL Medical Corporation, Chelmsford, MA, USA) \\
\hline Labetalol & Three Impact 326 suction machines (Impact Instrumentation, Inc.) \\
\hline Metoprolol & Three triple-channel IV pumps \\
\hline Furosemide & \\
\hline
\end{tabular}


assemble a standardized set and test it in exercises prior to use on patients. Aircraft noise impairs communication and interferes with the ability to rely on audible alarms on medical equipment. It is also common for the aircraft environment to interfere with the normal function of medical equipment, and it is possible for the medical equipment to interfere with safe operation of the aircraft. For this reason, all medical equipment used in air transport must be tested and certified for use in flight.

The physiologic aspects of flight impart unique stresses to the patient. The most obvious change is the decrease in ambient pressure to a typical altitude equivalent of 6,000 to 8,000 feet during long-range transport. Aircraft cabin altitude can be maintained near sea level, but this increases fuel consumption and limits aircraft range. Considerations for mechanical ventilation during long-range air transport have been reviewed [23]. Gas volume increases by a factor of 1.35 between sea level and 8,000 feet, so careful attention must be paid to trapped gas within the patient and within medical devices. Contraindications to air transport at reduced ambient pressure include decompression sickness and gas trapped in the thorax, pericardium, bowel, eye, or skull unless these conditions are specifically addressed. Gas expansion in an endotracheal tube cuff increases pressure on the tracheal mucosa, so air should be replaced with sterile saline or cuff pressure should be monitored and adjusted frequently during the transport. In spontaneously breathing patients, decreased partial pressure of oxygen is reflected in decreased arterial oxygen saturation unless oxygen supplementation is increased. The effect in mechanically ventilated patients is less obvious, likely due to the effectiveness of positive-end expiratory pressure in a hypobaric environment [24]. It has been the experience of CCATTs that nearly all patients with acute respiratory distress syndrome can be adequately oxygenated during long-range air transport while following a lung-protective strategy. The low humidity experienced during air transport causes increased insensible fluid loss in patients and caregivers. Patients with burns and tracheotomies as well as children and neonates are particularly susceptible to drying, so this must be accounted for in their care.

Acceleration causes complex physiologic changes, the net effects of which are difficult to predict. Patients likely to be highly susceptible to acceleration are those with severe left ventricular failure, increased ICP, and hypovolemic shock. In the absence of specific data, it is the authors' practice to position the patient so the vector of greatest anticipated acceleration runs perpendicular to the patient's long axis. In the example of a patient with increased ICP, the patient's torso is placed as upright as possible during takeoff and landing.

The major remaining challenges derive from the fact that a flying ICU does not have access to the capability present in a medical center. Foremost is rapid access to emergency surgical, angiographic, and endoscopic interventions. Portable blood analyzers are available, allowing basic chemistry, blood gas, and hemoglobin/hematocrit evaluation at the bedside. Diagnostic imaging in flight is currently possible only with portable ultrasound. This technology has an emerging role in critical care practice [25] and could advance the level of care available in flight. Expert consultation is possible using a telephone patch through the aircraft communication system, but this is not perfectly reliable. Transfusion support is available only if the requirement is identified before the flight and blood products, which may not be used, are committed to the patient. This will often pose a difficult choice in the locations from which critically ill patients are transferred. Despite these limitations, long-range critical care air transport is frequently performed.

\section{Conclusion}

The major means of providing critical care in austere locations are inserting portable critical care into the area of interest and transport of critically ill patients to a more capable location. There are military and civilian models for this care. These teams have a growing track record of success supporting military operations and providing relief in locations rendered austere by disaster. Further development of such teams at the local or regional level will make a significant contribution to the hospital-preparedness challenge facing the many communities with little or no ICU surge capacity. Portable ICUs can serve as a buffer for critically ill casualties as evacuation is being arranged and where needed until a more permanent capability is established.

The emerging capabilities to provide critical care in austere environments offer the most promise in the setting of response to a disaster, whether manmade or natural. Providing critical care in this setting raises complex sociopolitical issues. For certain disasters, the response timing is critical to ensure optimal casualty outcome. Local leaders must assess the threat to their community and address the degree to which they are willing to invest in preparedness. In this context, that means training and equipping critical care personnel to rapidly replace or augment community resources in the event of a crisis.

Recovery from critical illness or injury often requires extensive rehabilitative services and follow-up medical care. Planning for this care must begin immediately and should consider

\section{This article is part of a review series on Disaster management, edited by J Christopher Farmer.}

Other articles in the series can be found online at http://ccforum.com/articles/ theme-series.asp?series=CC_Disaster 
recovery of local resources and potential contribution relief agencies and non-governmental organizations. The combination of portable critical care with critical care transport appears promising in those disasters and military operations in which it has been employed. Data are beginning to emerge, and detailed study is required to determine whether the outcome of critically ill patients managed with this combination of capabilities meets expectations.

\section{Competing interests}

The authors declare that they have no competing interests.

\section{References}

1. Pronovost $\mathrm{P}$, Angus $\mathrm{D}$, Dorman $\mathrm{T}$, Robinson $\mathrm{K}$, Dremsizov $\mathrm{T}$, Young $T$ : Physician staffing patterns and clinical outcomes in critically ill patients. JAMA 2002, 288:2151-2162.

2. Wedel S, Warren J, Harvey M, Biel MH, Dennis R: Guidelines for intensive care unit design. Crit Care Med 1995, 23:582-588.

3. Cocanour C, Allen S, Mazabob J, Sparks J, Fischer C, Romans J, Lally K: Lessons learned from the evacuation of an urban teaching hospital. Arch Surg 2002, 137:1141-1145.

4. Nates J: Combined external and internal hospital disaster: impact and response in a Houston trauma center intensive care unit. Crit Care Med 2004, 32:686-690.

5. Farmer JC, Carlton PK: Providing critical care during a disaster: the interface between disaster response agencies and hospitals. Crit Care Med 2006, Suppl 34:56-59.

6. Rubinson L, Nuzzo JB, Talmor DS, O'Toole T, Kramer BR, Inglesby TV, for the Working Group on Emergency Mass Critical Care: Augmentation of hospital critical care capacity after bioterrorist attacks or epidemics: recommendations of the Working Group on Emergency Mass Critical Care. Crit Care Med 2005, Suppl 33:E2393.

7. Halpern P, Rosen B, Carasso S, Sorkine P, Wolf $Y$, Benedek P, Martinovich G: Intensive care in a field hospital in an urban disaster area: lessons from the August 1999 earthquake in Turkey. Crit Care Med 2003, 31:1410-1414.

8. D'Amore AR, Hardin CK: Air Force expeditionary medical support unit at the Houston floods: use of a military model in civilian disaster response. Mil Med 2005, 170:103-108.

9. Schnitzer JJ, Briggs SM: Earthquake relief-The U.S. medical response in Bam, Iran. N Engl J Med 2004, 350:1174-1176.

10. Klein KR, Nagel NE: Mass medical evacuation: Hurricane Katrina and nursing experiences at the New Orleans airport. Disaster Manag Response 2007, 5:56-61.

11. Maegele M, Gregor S, Steinhausen E, Bouillon B, Heiss M, Perbix W, Wappler F, Rixen D, Geisen J, Berger-Schreck B, Schwarz R: The long-distance tertiary air transfer and care of tsunami victims: injury pattern and microbiological and psychological aspects. Crit Care Med 2005, 33:1136-1140.

12. Centers for Disease Control and Prevention (CDC): Acinetobacter baumannii infections among patients at military medical facilities treating injured US service members, 2002-2004. MMWR Morb Mortal Wkly Rep 2004, 53:1063-1066.

13. Lopez M, Arnhold J: Safety of definitive in-theater repair of facial fractures. Arch Facial Plast Surg 2007, 9:400-405.

14. Briggs SM: Disaster management teams. Curr Opin Crit Care 2005, 11:585-589.

15. Murdock AD: Experience at the 332nd Air Force Theater Hospital: evacuation hub for Iraq. J Trauma 2007, Suppl 62:19.

16. Teichman $P$, Donchin $Y$, Kot R: International Aeromedical Evacuation. N Engl J Med 2007, 356:262-270.

17. Warren J, Fromm RE Jr., Orr RA, Rotello RC, Horst HM, American College of Critical Care Medicine: Guidelines for the inter- and intrahospital transport of critically ill patients. Crit Care Med 2004, 32:256-262.

18. Hetz SP: Introduction to military medicine: a brief overview. Surg Clin N Am 2006, 86:675-688.

19. Gawadne A: Casualties of war-military care for the wounded from Iraq and Afghanistan. N Engl J Med 2004, 351:2471-2475.

20. Grissom TE, Farmer JC: The provision of sophisticated critical care beyond the hospital: lessons from physiology and mili- tary experiences that apply to civil disaster medical response. Crit Care Med 2005, Suppl 33:13-21.

21. Young SHH: Gallery of USAF weapons. Air Force Magazine 2007, 90:146.

22. Air Mobility Command Public Affairs Office: U.S. Air Force Fact Sheet: C-17 Globemaster III. Scott Air Force Base, IL: Air Mobility Command Public Affairs Office; May 2006.

23. Beninati $W$, Jones KD: Mechanical ventilation during long range air transport. Respir Care Clin 2002, 8:51-65.

24. Lawless N, Tobias S, Mayorga MA: $\mathrm{FIO}_{2}$ and positive end-expiratory pressure as compensation for altitude-induced hypoxemia in an acute respiratory distress syndrome model: implications for air transportation of critically ill patients. Crit Care Med 2001, 29:2149-2155.

25. Kirkpatrick AW, Sustic A, Blaivas M: Introduction to the use of ultrasound in critical care medicine. Crit Care Med 2007 , Suppl 35:S123-125. 